\title{
Estrategia de mecanizado óptima en operaciones de fresado 3D en superficies inclinadas, con alta velocidad de corte (HSM) en el aluminio 7075 T6
}

\author{
Xavier Vaca, Borys Culqui \\ Centro de Posgrados, Universidad de las Fuerzas Armadas ESPE \\ xvaca@ups.edu.ec, bhculqui@espe.edu.ec
}

\begin{abstract}
Resumen-En el presente proyecto de investigación, se ha seleccionado cuatro tipos de estrategias de fresado $3 D$ de acabado para el mecanizado en superficies inclinadas, el material de trabajo es aleación de aluminio 7075 T6 y se analizó la calidad superficial a través de la medición de la rugosidad. En los ensayos se varía la velocidad de corte y se mantiene constante los parámetros de corte como velocidad de avance, profundidad de corte, paso y velocidad del husillo conforme al recomendado por el fabricante de la fresa punta redonda. La velocidad de corte varía a lo largo del filo de la herramienta esférica. Se realizó un diseño CAD con ocho pendientes, de $35^{\circ}$ a $87,5^{\circ}$ en intervalos de $7,5^{\circ}$. Se utilizó una metodología experimental, donde se combinó todas las variables, por lo tanto las ocho superficies fueron mecanizadas en seco y evaluadas por cada estrategia. Se realizó un análisis de resultados y se encontró que la estrategia que presenta menor rugosidad superficial es la de paralelo con trayectoria en descenso (PD), con un rango de velocidad de corte efectiva de 260 a $422 \mathrm{~m} / \mathrm{min}$ y se obtuvo una rugosidad Ra promedio de $0,295 \mu \mathrm{m}$.
\end{abstract}

Palabras Claves-Estrategias de mecanizado, Mecanizado de alta velocidad HSM, Rugosidad superficial, trayectoria en descenso.

\begin{abstract}
This research project establishes four different strategies of 3D end milling for inclined surfaces. The workpiece material used in the project was aluminum alloy 7075 T6. The aim of the project analyzes the surface quality of the product in each strategy. The process has fixed four parameters: feed speed, depth of cut, width of cut and spindle speed according to the tool manufacturer. A ball nose end mills was use in the process. The cutting speed varies along the edge of the spherical tool. The CAD design has eight steps between $35^{\circ}$ to $87,5^{\circ}$ in gaps of $7,5^{\circ}$. The experimental methodology was applied. The eight surfaces were dry machined and evaluated by each strategy. An analysis of results was carried out and it was found that the strategy that presents the lowest surface roughness is that of parallel with a downward trajectory (PD), with an effective cutting speed range of 260 to $422 \mathrm{~m} / \mathrm{min}$ and a roughness Ra was obtained. average of $0.295 \mu \mathrm{m}$.
\end{abstract}

Keywords- Strategies of machining, High speed machining HSM, Surface roughness, downward milling

\section{INTRODUCCIÓN}

El desarrollo de investigaciones y experimentos en operaciones de mecanizado en aleaciones de aluminio 7075 T6, tiene un gran interés en la industria de moldes y matrices debido a su alta resistencia mecánica y bajo peso [1]. En algunos artículos concernientes con el mecanizado, realizan ensayos con variaciones en los parámetros de corte y mencionan algunos criterios: En un estudio [2], menciona que, para el mecanizado en superficies con inclinaciones, la velocidad de corte varía alrededor del filo de la fresa punta esférica en el punto de contacto con la superficie y la trayectoria que ésta realiza, ya sea en ascenso o en descenso. $\mathrm{Y}$ propone que se obtienen valores de rugosidad pequeños con altas velocidades de corte en este caso $188 \mathrm{~m} / \mathrm{min}$.

En cuanto a trayectorias de mecanizado [3], determina, que el fresado con trayectoria en ascenso, presenta mayor estabilidad de corte y menor flexión de la herramienta, sin embargo, no presenta resultados de calidad superficial.

La primera definición de altas velocidades de corte HSM fue propuesta por el Dr. Ing. Carl Salomon en 1931, su argumento se basaba en que la temperatura de corte alcanzaba un pico a una velocidad de corte determinada, en el caso de materiales no ferrosos, la velocidad critica es de $200 \mathrm{~m} / \mathrm{min}$ y una temperatura de $900{ }^{\circ} \mathrm{C}$, sin embargo, si se seguía aumentando esta velocidad, la temperatura de corte disminuía [4], ver figura 1, por lo tanto, trabajar con altas velocidades de corte, puede ofrecer varias ventajas con relación al mecanizado con velocidad convencional.

Un estudio presentado en [5], menciona que la velocidad de tiene un efecto dominante en la rugosidad superficial, y que esta disminuye cuando la velocidad de corte aumenta.

El presente proyecto desea apreciar el comportamiento de la rugosidad Ra en superficies inclinadas mecanizadas con altas velocidades de corte, desde 160 a $440 \mathrm{~m} / \mathrm{min}$, con cuatro diferentes estrategias de mecanizado y determinar las condiciones de velocidad de corte, trayectoria y dirección de la herramienta, para obtener una mejor calidad superficial.

\section{MÉTODO}

\section{A. Descripción del experimento}

El material de las probetas mecanizadas es AA7075 T6. La herramienta para el acabado es una fresa de carburo revestido TiAlN, diámetro $14 \mathrm{~mm}, 4$ filos, marca Izartool. Se utilizó un centro de mecanizado CNC de tres ejes marca EMCO con control Sinumerik. El mecanizado se realizó sin refrigerante.

La velocidad de corte fue de 160 a $440 \mathrm{~m} / \mathrm{min}$. Se considera HSM según las curvas del Dr Salomon, expuestas en la Fig 1. Para materiales no ferrosos, la velocidad de corte crítica es $200 \mathrm{~m} / \mathrm{min}$ y superior a este valor se trabaja con HSM, donde la temperatura de mecanizado empieza a descender a medida que la velocidad de corte aumenta [4].

\section{B. Modelo $C A D$}

El diseño contiene superficies con diferentes pendientes, en total ocho, en intervalos de $7.5^{\circ}$ y son: $35^{\circ} / 42.5^{\circ} / 50^{\circ} /$ $57.5^{\circ} / 65^{\circ} / 72.5^{\circ} / 80^{\circ} / 87.5^{\circ}$. 


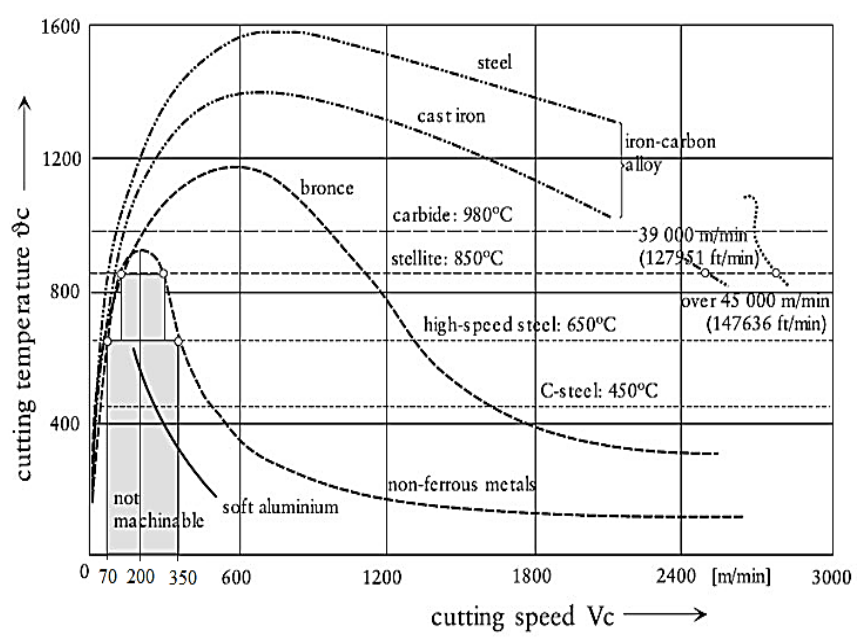

Fig. 1. Curvas de Salomon [4].

\section{Estrategias de mecanizado y trayectorias}

La programación se realizó en un software de gama alta, las estrategias 3D que se definieron para el proceso de acabado son: Contorno (z constante) y Paralelo (z no constante), con trayectoria en ascenso y en descenso como se muestra en la Fig. 2.

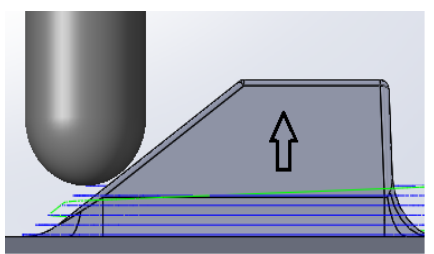

a)

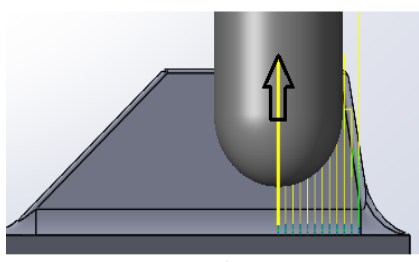

c)

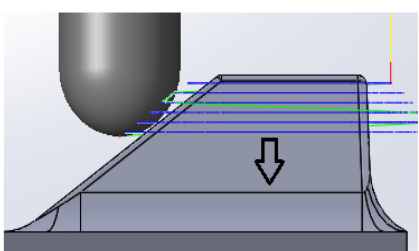

b)

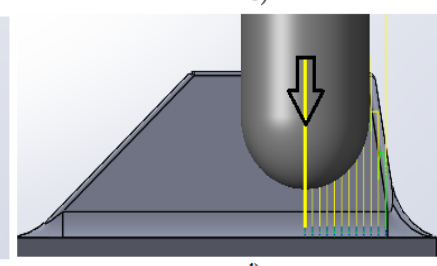

d)
Fig. 2. Estrategias (a) Contorno en ascenso CA, (b) Contorno de descenso CD, (c) Paralelo en ascenso PA, (d) Paralelo en descenso PD.

\section{Parámetros de corte}

Los parámetros de corte que se mantienen constantes conforme al recomendado por el fabricante de la fresa en el catálogo [6] son $\mathrm{Fz}=0.05 \mathrm{~mm} / \mathrm{z}, \mathrm{Vf}=2000 \mathrm{~mm} / \mathrm{min}$, ap y $a e=0.2 \mathrm{~mm}, N=10000 \mathrm{RPM}$

La velocidad de corte efectiva $\left(V_{C e f}\right)$ y el diámetro efectivo $\left(D_{e f}\right)$ dependen de la pendiente, trayectoria y profundidad de corte ap, como muestra la Fig. 3.

El diámetro efectivo en ascenso se obtiene con la expresión proveniente de la referencia [7]:

$$
D_{e f a}=D \cdot \sin \left[\alpha+\operatorname{arcos}\left(\frac{D-2 a p}{D}\right)\right]
$$

La velocidad de corte efectiva se obtiene con la expresión proveniente de la referencia [8]:

$$
V_{C e f}=\frac{\pi \cdot N \cdot D_{e f}}{1000}
$$

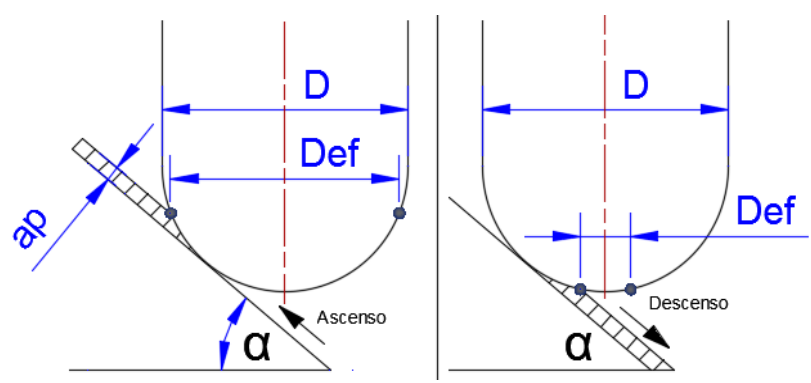

Fig. 3. Relación de la pendiente y diámetro efectivo con a) trayectoria en ascenso, b) trayectoria en descenso.

Con trayectoria en descenso, el diámetro efectivo es menor, se obtiene con la expresión:

$$
D_{e f d}=D \cdot \sin \left[\alpha+\operatorname{arcos}\left(\frac{D-2 a p}{D}\right)\right]
$$

La variación del diámetro efectivo con herramienta de diámetro 14 mm se muestra en la Fig. 4.

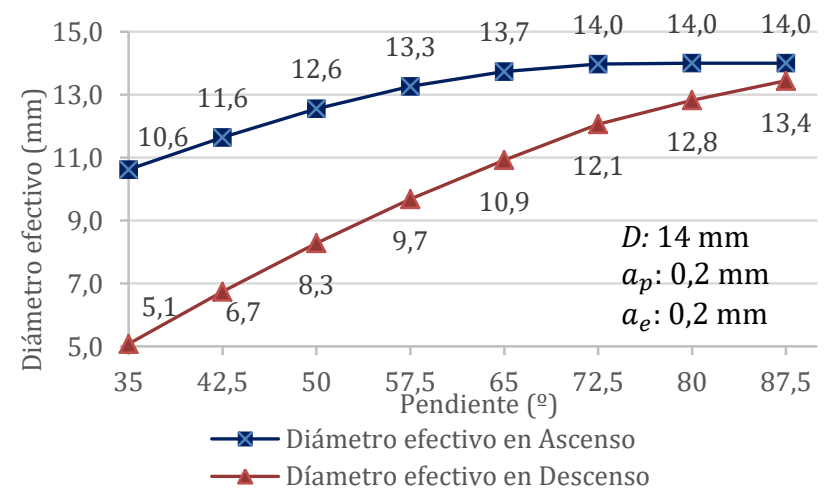

Fig. 4. Relación entre diámetro efectivo y pendiente.

Esto exige a calcular la velocidad de corte en función del diámetro efectivo.

\section{E. Detalle del experimento.}

El experimento tiene un diseño que combina todas las variables, en la tabla 1 , se muestra el conjunto de pruebas que se realizaron.

\section{F. Medición de la rugosidad superficial.}

La medición de la rugosidad se realizó en sentido perpendicular al mecanizado con el rugosímetro Mitutoyo SJ210, se realizan treinta y dos experimentos, con tres muestras de cada una, es decir se mecanizan y se miden en total noventa y seis superficies.

\section{ANÁLISIS DE RESUlTAdOS}

El análisis de la calidad superficial se realizó a través de la medición de rugosidad en las superficies mecanizadas. 
TABLA I

DETALLE DE EXPERIMENTOS

\begin{tabular}{|c|c|c|c|c|c|}
\hline \multicolumn{4}{|c|}{$\begin{array}{l}\text { Velocidad Husillo: } \\
\text { RPM } \\
\text { RP: } \\
\text { ap: } \quad 0,2 \mathrm{~mm} \\
\text { ae: } \quad 0,2 \mathrm{~mm} \\
\end{array}$} & \multicolumn{2}{|c|}{$\begin{array}{l}\text { D: } 14 \mathrm{~mm} \\
\text { Vf: } 2000 \\
\text { F: } 0,05 \mathrm{~mm} / \mathrm{z}\end{array}$} \\
\hline No & Exp. & $\begin{array}{l}\text { Estrategia } \\
\text { / } \\
\text { Trayectori }\end{array}$ & $\begin{array}{c}\text { Pendiente } \\
{\left[{ }^{\circ}\right]}\end{array}$ & $\begin{array}{c}\text { Def } \\
{[\mathrm{mm}]}\end{array}$ & $\begin{array}{c}\text { Vcef } \\
{[\mathbf{m} / \mathbf{m i}} \\
\text { n] }\end{array}$ \\
\hline 1 & $\begin{array}{l}\text { CA } 1 \\
\text { CA } 2 \\
\text { CA } 3 \\
\text { CA } 4 \\
\text { CA } 5 \\
\text { CA } 6 \\
\text { CA } 7 \\
\text { CA } 8 \\
\end{array}$ & $\begin{array}{l}\text { Contorno / } \\
\text { Ascenso } \\
\text { (CA) }\end{array}$ & $\begin{array}{c}35 \\
42,5 \\
50 \\
57,5 \\
65 \\
72,5 \\
80 \\
87,5 \\
\end{array}$ & $\begin{array}{l}10,6 \\
11,6 \\
12,6 \\
13,3 \\
13,7 \\
14,0 \\
14,0 \\
14,0 \\
\end{array}$ & $\begin{array}{l}333 \\
365 \\
394 \\
416 \\
431 \\
439 \\
440 \\
440 \\
\end{array}$ \\
\hline 2 & $\begin{array}{l}\text { PA } 1 \\
\text { PA } 2 \\
\text { PA } 3 \\
\text { PA } 4 \\
\text { PA } 5 \\
\text { PA } 6 \\
\text { PA } 7 \\
\text { PA } 8 \\
\end{array}$ & $\begin{array}{l}\text { Paralelo / } \\
\text { Ascenso } \\
\text { (PA) }\end{array}$ & $\begin{array}{c}35 \\
42,5 \\
50 \\
57,5 \\
65 \\
72,5 \\
80 \\
87,5 \\
\end{array}$ & $\begin{array}{l}10,6 \\
11,6 \\
12,6 \\
13,3 \\
13,7 \\
14,0 \\
14,0 \\
14,0 \\
\end{array}$ & $\begin{array}{l}333 \\
365 \\
394 \\
416 \\
431 \\
439 \\
440 \\
440 \\
\end{array}$ \\
\hline 3 & $\begin{array}{l}\text { CD } 1 \\
\text { CD } 2 \\
\text { CD } 3 \\
\text { CD } 4 \\
\text { CD } 5 \\
\text { CD } 6 \\
\text { CD } 7 \\
\text { CD } 8 \\
\end{array}$ & $\begin{array}{l}\text { Contorno / } \\
\text { Descenso } \\
\text { (CD) }\end{array}$ & $\begin{array}{c}35 \\
42,5 \\
50 \\
57,5 \\
65 \\
72,5 \\
80 \\
87,5 \\
\end{array}$ & $\begin{array}{c}5,1 \\
6,7 \\
8,3 \\
9,7 \\
10,9 \\
12,1 \\
12,8 \\
13,4 \\
\end{array}$ & $\begin{array}{l}160 \\
212 \\
260 \\
304 \\
343 \\
379 \\
403 \\
422 \\
\end{array}$ \\
\hline 4 & $\begin{array}{l}\text { PD1 } \\
\text { PD2 } \\
\text { PD3 } \\
\text { PD4 } \\
\text { PD5 } \\
\text { PD6 } \\
\text { PD7 } \\
\text { PD8 } \\
\end{array}$ & $\begin{array}{l}\text { Paralelo / } \\
\text { Descenso } \\
\text { (PD) }\end{array}$ & $\begin{array}{c}35 \\
42,5 \\
50 \\
57,5 \\
65 \\
72,5 \\
80 \\
87,5 \\
\end{array}$ & $\begin{array}{c}5,1 \\
6,7 \\
8,3 \\
9,7 \\
10,9 \\
12,1 \\
12,8 \\
13,4 \\
\end{array}$ & $\begin{array}{l}160 \\
212 \\
260 \\
304 \\
343 \\
379 \\
403 \\
422 \\
\end{array}$ \\
\hline
\end{tabular}

\section{A. Comparación de rugosidad por estrategias}

En la Fig. 5 se muestra un diagrama de dispersión con líneas suavizadas de los valores de la rugosidad superficial Ra obtenidos con las estrategias en cada pendiente. La mejor rugosidad promedio entre las cuatro estrategias mecanizadas es la que se obtiene con estrategia de (PD).

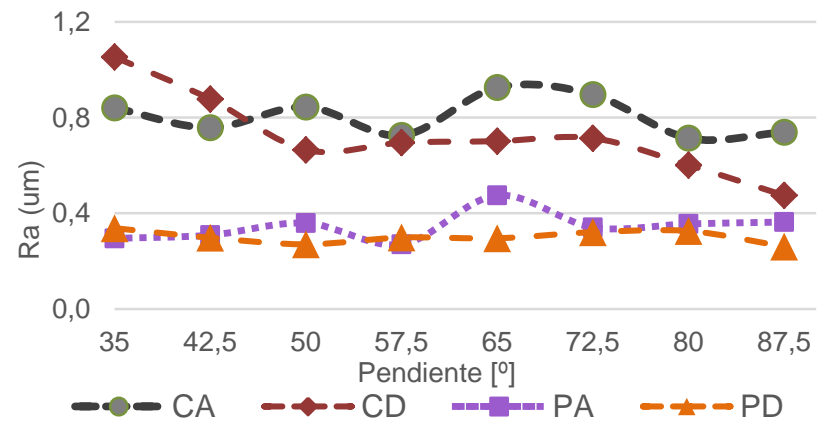

Fig. 5. Comparación de la rugosidad Ra obtenida con las diferentes estrategias en cada pendiente.

\section{B. Rugosidad promedio $R a, R q, R z, R$ th}

En el proceso de medición de rugosidad superficial, se obtuvo la rugosidad media aritmética Ra, rugosidad media cuadrática $R q$ y la profundidad media de rugosidad $R z$.

La rugosidad teórica o altura de la cresta Rth, se obtuvo con la ecuación número 5 proveniente de la referencia [9], para estrategias de contorno con dirección z constante:

$$
R_{t h}=R-\sqrt{R^{2}-\left(\frac{a_{p}}{2 \cdot \sin \alpha}\right)^{2}}
$$

donde $R$ es el radio de la herramienta esférica y $\alpha$ es el ángulo de la pared.

La ecuación 6 para estrategias de paralelo con dirección $z$ no constante:

$$
R_{t h}=\frac{a_{e}^{2}}{8 \cdot R}
$$

En la Tabla II se muestra los resultados de la rugosidad superficial promedio Ra, Rq, Rz y Rth con cada estrategia mecanizada.

TABLA II

\begin{tabular}{|c|c|c|c|c|}
\hline $\begin{array}{l}\text { Estrategia/ } \\
\text { Trayectoria }\end{array}$ & $\begin{array}{r}\text { Prom } \\
\text { Ra }[\mu \mathrm{m}]\end{array}$ & $\begin{array}{c}\text { Prom } \\
\text { Rq } \\
{[\mu \mathrm{m}]}\end{array}$ & $\begin{array}{l}\text { Prom } \\
\text { Rz } \\
{[\mu \mathrm{m}]} \\
\end{array}$ & $\begin{array}{c}\text { Prom } \\
\text { Rth }[\mu \mathrm{m}]\end{array}$ \\
\hline CA & 0,805 & 0,969 & 4,386 & 1,133 \\
\hline CD & 0,723 & 0,875 & 3,956 & 1,133 \\
\hline PA & 0,346 & 0,416 & 1,682 & 0,714 \\
\hline PD & 0,301 & 0,364 & 1,513 & 0,714 \\
\hline
\end{tabular}

RUGOSIDAD SUPERFICIAL PROMEDIO

\section{Rugosidad Ra promedio por velocidad de corte}

En la Fig. 6, se muestra el comportamiento de la rugosidad promedio con respecto a la velocidad de corte efectiva desde 160 a $440 \mathrm{~m} / \mathrm{min}$. La rugosidad disminuye a medida que la velocidad de corte aumenta hasta una velocidad de $260 \mathrm{~m} / \mathrm{min}$, a partir de este punto la curva de la rugosidad varia con menor regularidad, hasta que a 422 $\mathrm{m} / \mathrm{min}$ se obtiene la menor rugosidad.

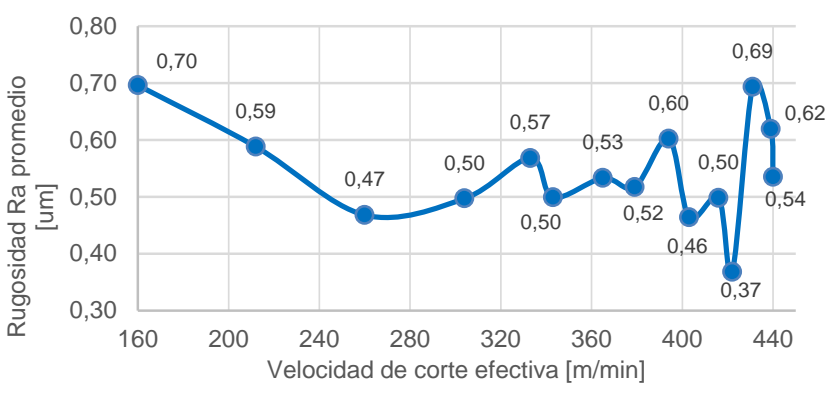

Fig. 6. Velocidad de corte efectiva vs rugosidad.

\section{Análisis de trayectoria}

Para estrategias de contorno y paralelo se tiene una rugosidad promedio menor con trayectorias en descenso como se muestra en la Fig. 7.

\section{E. Análisis de estrategia}

La estrategia que obtiene una menor rugosidad es la de paralelo, la rugosidad disminuye en un 57,8 \% con relación a la estrategia de contorno, como se ve en la figura 8 . 


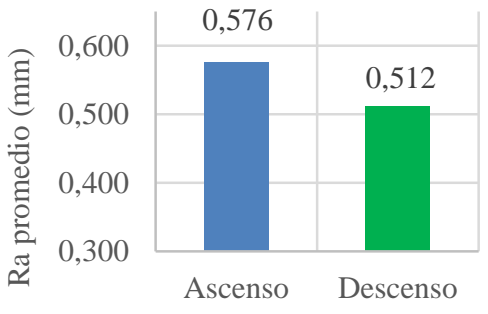

Fig. 7. Trayectoria con menor rugosidad.

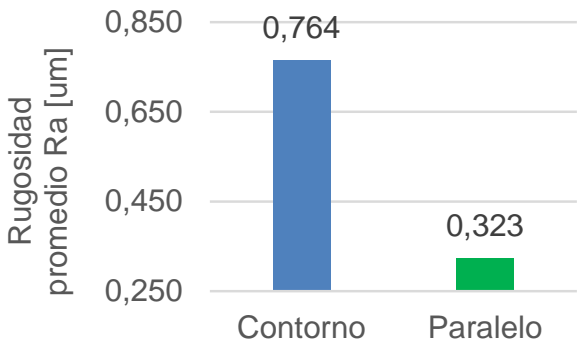

Fig. 8. Estrategia con menor rugosidad.

\section{F. Estrategia de mecanizado óptima}

En la Tabla III, se muestra los resultados finales del análisis que permiten proponer una estrategia de mecanizado óptima.

TABLA III

RESULTADOS PARA UNA ESTRATEGIA ÓPTIMA

\begin{tabular}{cc}
\hline \hline Condiciones & Resultado óptimo \\
\hline $\begin{array}{c}\text { Velocidad de corte } \\
\text { efectiva }\end{array}$ & $422[\mathrm{~m} / \mathrm{min}]$ \\
Trayectoria & Descenso \\
Estrategia & Paralelo \\
\hline \hline
\end{tabular}

La velocidad de corte varía con las pendientes, de modo que, no es posible fijar una velocidad de corte para toda una superficie $3 \mathrm{D}$, por esto se establece un rango de velocidad.

\section{G. Resultados del Análisis}

En la Tabla IV se muestra los mejores resultados del análisis para cada estrategia, en condiciones que permitan una menor rugosidad superficial.

TABLA IV

RESULTADOS ÓPTIMOS DE CADA ESTRATEGIA.

\begin{tabular}{ccccc}
\hline \hline $\mathbf{N}$ & $\begin{array}{c}\text { Estrategia } \\
\text { Trayectoria }\end{array}$ & Pendiente $\left[^{\circ} \mathbf{]}\right.$ & $\begin{array}{c}\text { Vcef. } \\
{[\mathbf{m} / \mathbf{m i n}]}\end{array}$ & $\begin{array}{c}\text { Ra prom } \\
{[\boldsymbol{\mu m}]}\end{array}$ \\
\hline 1 & $(\mathrm{CA})$ & $35^{\circ}$ a $57,5^{\circ}$ & 333 a 416 & 0,791 \\
2 & $(\mathrm{CD})$ & $50^{\circ}$ a $87,5^{\circ}$ & 260 a 422 & 0,641 \\
3 & $(\mathrm{PA})$ & $35^{\circ}$ a $57,5^{\circ}$ & 333 a 416 & 0,308 \\
4 & $(\mathrm{PD})$ & $50^{\circ}$ a $87,5^{\circ}$ & 260 a 422 & 0,295 \\
\hline \hline
\end{tabular}

\section{H. Resultados óptimos de cada estrategia.}

La Fig. 9 muestra el rango de velocidad de corte óptimo para cada estrategia.

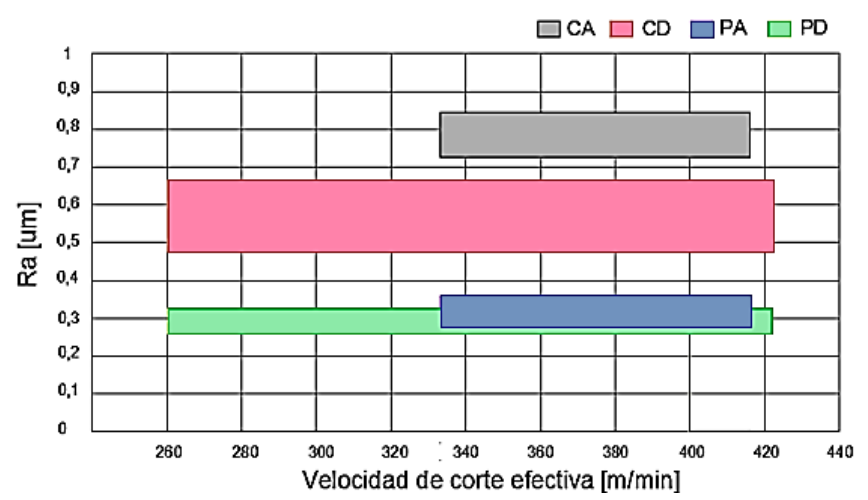

Fig. 9. Rango de velocidad de corte óptimo.

\section{CONCLUSIONES}

Trayectorias en descenso reduce la rugosidad en un 11,1 \% con respecto a la que se obtiene con trayectorias en ascenso. Estrategia de paralelo reduce la rugosidad en un $57,8 \%$ con respecto a la que se obtiene con estrategia de contorno. La estrategia de mecanizado que muestra una menor rugosidad es la (PD), con un rango de velocidad de corte efectiva entre $260 \mathrm{~m} / \mathrm{min}$ a $422 \mathrm{~m} / \mathrm{min}$, que presenta un valor de rugosidad promedio Ra de 0,295 $\mu \mathrm{m}$ como se mostró en la tabla 4.

\section{REFERENCIAS}

[1] Ivan Bohman, «Duraluminio para moldes plásticos catálogo,» Ivan Bohman, Quito, 2015.

[2] Lemes Capla, «Efectos de la variación de sobremedida debido al uso de 2 1 12 ejes en la operación de acabado.,» Ingeniería Mecánica, vol. 1 , pp. 31 - 39, 2008.

[3] M. Lee a, «The optimal cutter orientation in ball end milling of cantilever-shaped thin plate,» Material Processing Technology, vol. 1, pp. 900 - 906, 2004.

[4] L. M. López de Lacalle Marcaide, Mecanizado de Alto Rendimiento, 1st ed., Bilbao: Izaro, 2004.

[5] P. L. \&. S. B. Pasko, «High speed machining (HSM) - The effective way of modern cutting,» de International Workshop CA Systems And Technologies, Poland, 2002.

[6] IzarTool, «Catálogo de parámetros de corte de herramientas IzarTool,» IzarTool, España, 2017.

[7] M. J. Wang, «Residual Stresses Analysis in Ball end Milling of Nickel-Based Superalloy Inconel 718,» Materials Reserch, vol. 20, pp. 1681 - 1689, 2017.

[8] S. S Kalpakjian S R, [1] S. G. López de Lacalle Marcaide, Lamikiz Menchaca, Mecanizado de Alto Rendimiento, 1st ed. Bilbao: Izaro, 2004., México: Pearson, 2016.

[9] M. Balázs, «Study of Z-level Finishing Milling Strategy,» Development in machining technology, vol. 2, pp. 83 - 90, 2012. 